\title{
Correction to: Mechanical activation of Ti02/Fe203 nanocomposite for arsenic adsorption: effect of ball-to-powder ratio and milling time
}

\author{
Mercyrani Babudurai ${ }^{1}$ Onyekachi Nwakanma ${ }^{2} \cdot$ Araceli Romero-Nuñez $^{2} \cdot$ Ravichandran Manisekaran $^{3}(1) \cdot$ \\ Velumani Subramaniam ${ }^{1,2}$. Homero Castaneda ${ }^{4} \cdot$ Anish Jantrania $^{5}$
}

Published online: 29 September 2021

(c) Islamic Azad University 2021

\section{Correction to: Journal of Nanostructure in Chemistry https://doi.org/10.1007/s40097-021-00388-8}

The author, Dr. Velumani Subramaniam request to remove the below affiliation as there was a travel restrictions and administrative delays and he was not able to realize the sabbatical and by mistake left the affiliation.

Department of Materials Science and Engineering, Texas A\&M University, College Station, TX, 77,843, USA.

The Original article was corrected.

Publisher's Note Springer Nature remains neutral with regard to jurisdictional claims in published maps and institutional affiliations.

The original article can be found online at https://doi.org/10.1007/ s40097-021-00388-8.

Velumani Subramaniam

velu@ cinvestav.mx

1 Nanoscience and Nanotechnology Program, Centro de Investigación y de Estudios Avanzados del Instituto Politécnico Nacional (CINVESTAV-IPN), Av. Instituto Politécnico Nacional 2508, Col. San Pedro Zacatenco, Ciudad de México 07360, Mexico

2 Department of Electrical Engineering (SEES), Centro de Investigación y de Estudios Avanzados del Instituto Politécnico Nacional (CINVESTAV-IPN), Av. Instituto Politécnico Nacional 2508, Col. San Pedro Zacatenco, 07360 Ciudad de México, Mexico

3 Laboratorio de Investigación Interdisciplinaria, Área de Nanoestructuras y Biomateriales, Escuela Nacional de Estudios Superiores Unidad León; Universidad Nacional Autónoma de México, León, Guanajuato, Mexico

4 Department of Materials Science and Engineering, Texas A\&M University, College Station, TX 77843, USA

5 Department of Biological and Agricultural Engineering, Agrilife Extension, Texas A\&M University, College Station, TX 77843, USA 